Rita Braches-Chyrek

Charlotte Röhner

Heinz Sünker

Michaela Hopf (Hrsg.)

\title{
Handbuch Frühe Kindheit
}

2., aktualisierte und erweiterte Auflage

Verlag Barbara Budrich

Opladen • Berlin • Toronto 2020 


\section{Constanze Kirchner}

\section{Ästhetische Bildung in der frühen Kindheit}

Bildungspläne für den Elementarbereich sind seit einigen Jahren in nahezu allen Ländern etabliert. Die ästhetische Bildung spielt dabei eine zentrale Rolle: Zwar mit verschiedenen Begriffen versehen, liegen doch die Schulung der Wahrnehmung, das spielerische Lernen mit allen Sinnen, der gestalterische Ausdruck - ob mit dem Körper, beim Bauen oder mit Stiften auf dem Papier im Blickpunkt der Bildungsprogramme. Das bildnerische Gestalten ist die am häufigsten genutzte Bezeichnung für jene Dimension ästhetischer Bildung, die speziell bildnerisch-gestaltende Aktivitäten und den Umgang mit Bildern bzw. Kunstwerken fokussiert (www.gew.de/bildungsplaene.html). Kinder sind neugierig und wissensdurstig, sie untersuchen, ordnen und gestalten aktiv ihr Umfeld, um sich zu orientieren und auszudrücken. Etwas Gestaltetes hervorzubringen hat immer mit Selbstvergewisserung zu tun: Eine Spur zu hinterlassen bedeutet, etwas zu verursachen, einen Ort zu markieren. Das Schmieren und Kritzeln bringt dem Kind nicht nur Befriedigung, sondern auch Selbstsicherheit am sichtbaren Erfolg. Wenn kleine Kinder im Sand eine Linie ziehen, kommt es vor, dass sie fasziniert auf das selbst erzeugte Zeichen schauen. Sie wiederholen den Vorgang, z. B. mit der Hand eine Spur zu hinterlassen, unzählige Male. Zweckfrei Spuren zu erzeugen, also aktiv gestaltend in die Umwelt einzugreifen, ist seit jeher in der Menschheitsgeschichte verankert.

\section{Dimensionen ästhetischer Bildung}

Ästhetische Bildung entsteht im Wechselspiel von Rezeption und Produktion in den Bereichen Musik, Tanz, Bildende Kunst, Literatur, Poesie. Der Rezeptionsprozess - z. B. beim Betrachten von Gemälden - ist keine passive Angelegenheit, sondern erfordert eigenständiges Sinnerschließen. Kinder gestalten Bilder und Kunstwerke aktiv mit - ästhetische Phänomene bieten die Möglichkeit, sich selbst in das Werk mit allen Empfindungen und Gedanken einzubringen, eigene Vorstellungen dazu zu entwickeln und eigene Interpretationen mit Mut vorzutragen (Schäfer 2004: 117 ff.). Im Produktionsprozess zeigt sich die aktive Gestaltung von Wirklichkeit offensichtlicher: Das Musizieren, Tanzen, Gedichte verfassen, Zeichnen, Malen, Bauen oder Konstruieren erfordert das Aktivieren innerer Vorstellungen, innerer Klänge, innerer Dialoge, innerer Bilder, Fantasien und Träume. Es wird etwas hervorgebracht - und zwar etwas, das auf ästhetische Weise gestaltet ist. Mit dieser Hervorbringung verbinden sich mehrere Aspekte: Einerseits entstehen Kompetenzgefühle, wenn etwas hervorgebracht wird, und damit wächst das Selbstwertgefühl, die Ich-Stärke. Andererseits wird etwas ausgedrückt - ein Erlebnis, eine Erinnerung, eine Vorstellung. Kinder drücken sich recht unterschiedlich aus: zeichnerisch oder mit Farbe, dreidimensional, mit Tönen und Klängen, gestisch, mimisch, mit dem ganzen Körper.

Insbesondere das bildnerisch-praktische Tun stellt eine Brücke zwischen dem Innen der inneren Vorstellungs-, Fantasie- und Traumwelt - und dem Außen - also eine Brücke zur äußeren Wirklichkeit her. In diesem Prozess, etwas von Innen nach Außen zu bringen und ihm in ästhetischer Form Gestalt zu verleihen, wird geordnet, strukturiert und gegliedert, um zu einer Form zu finden. Und innerhalb dieses Formfindungsprozesses wird das Erlebte verarbeitet - in der Auseinandersetzung mit dem Selbst und dem Anderen. Diese Leistung, eine Vorstellung wie auch immer geordnet - mit Begriffen oder zeichnerisch - auf das Papier zu 
bringen, zu modellieren oder körperlich darzustellen, ist ein vielschichtiger kreativer Akt, der das Verhältnis zur Welt ein Stück weit zu klären vermag. Das Erlebte wird organisiert, strukturiert und symbolisch ausgedrückt. Zugleich wird das Hervorgebrachte auf diese Weise zu einem Gegenüber, das mit Distanz betrachtet und reflektiert werden kann (Kirchner 2008). Zugleich werden durch den Umgang mit verschiedenen Materialien die Entwicklungsgrundlagen im triebdynamischen, emotionalen und sinnlichen Bereich, die zum Aufbau kognitiver Kompetenzen Voraussetzung sind, gefördert. Amorphe Materialien wie Sand, Wasser, Ton lösen im direkten Kontakt der Hände taktile Sinnesreize aus, lassen spontane Gestaltungen zu und können wegen der leichten Formbarkeit motivierend auch für gehemmte und scheue Kinder wirken, diese aus der Reserve locken und die Aktivität steigern. Weiche Materialien sind geeignet, um Spannungen schnell abzubauen, können jedoch auch chaotisches Entladen mit Kontrollverlusten auslösen. Harte Materialien wie Holz oder Stein hingegen bieten Widerstände, die der Aggressionsabfuhr dienen können (Wichelhaus 1995).

Das genaue Beobachten und Beschreiben von Bildern oder Kunstwerken dient nicht nur der Sprachförderung, sondern schärft die Wahrnehmung für gestalterische Merkmale wie Farbwirkungen, Kontraste und Kompositionen.

\section{Vorstellungen bilden - Wirklichkeit verarbeiten}

Vorstellungen, also innere Bilder, sind Grundlage des Denkens. Ohne eine Vorstellung kann kein Begriff gebildet werden. Die Ausbildung vielfältiger Ideen und Lösungsansätze wird geschwächt, wenn nicht durch eigene Kraft innere Bilder erzeugt und geäußert werden - wie die ästhetische Bildung es trainiert. Bei allen ästhetischen Tätigkeiten wie Sammeln und Ordnen, Tanzen und Singen, Malen und Zeichnen, Bauen und Konstruieren werden Wirklichkeitsszenarien entworfen, die die Identitätsbildung fördern. Es eröffnen sich Möglichkeiten zum Anderssein, zum Probehandeln und zur Antizipation von Wirklichkeit. Die Identitätsentwicklung wird in besonderem Maße durch die Differenzierung sinnlicher Eindrücke, die Reflexion und die Selbstwahrnehmung unterstützt. Hierbei hilft das Gestalten, weil Bilder, Symbole und Vorstellungen dabei vertieft und erweitert werden. Nicht allein der Wissenserwerb ist das entscheidende Element für späteren beruflichen Erfolg, sondern vielmehr ein breites Netz von Denkmöglichkeiten, das sich im Spiel, durch Erzählungen, Exkursionen, Entdeckungen in der Natur entfaltet. Freies Spiel sowie die komplexe Bildung mit Literatur, Musik und Kunst, die gedankenreiche Interpretationsübungen beinhaltet, sollen, so Rittelmeyer (2007), dazu beitragen, Imaginations- und Kreativitätsvorstellungen auszubilden. Je vielfältiger die Anregungen und Impulse für die Kinder sind, desto komplexere Denkstrukturen entwickeln sich. Zugleich muss den Kindern jedoch Zeit gegeben werden, ihre Erlebnisse zu filtern, zu sortieren, Ordnungen und Kategorien zu bilden, damit sie nicht in der Flut sinnloser Daten untergehen. Das bedeutet konkret: Sie brauchen die Möglichkeit, ihre Welt zu erkunden, zu experimentieren, sinnliche Erfahrungen zu machen etc. und zugleich sehr viel Zeit, diese Erlebnisse angemessen im Spiel, im Erzählen, im bildnerischen Gestalten zu verarbeiten. Gerade das gestalterische Tun trägt in hohem Maße zur Integration und Ordnung des Erfahrenen bei, weil der Verarbeitungs- und Formfindungsprozess verlangsamt und intensiviert vonstatten geht (Kirchner 2008).

Die Persönlichkeitskonstruktion wird von einem dynamischen Einpassen sinnlicher Erfahrungen und ästhetischer Wahrnehmungen in das Selbstbild begleitet und im steten Austausch mit dem Innen und dem Außen entwickelt, unterstützt durch die gestalterische Verarbeitung. Das bildnerische Tun und die Sprache sind dabei elementare Strukturierungsmittel, 
die das Erlebte in sinnvolle Ordnungen bringen. Der Kognitionspsychologe Howard Gardner weist darauf hin, dass Kinder über zahlreiche Intelligenzen verfügen, die allesamt gleichermaßen zu fördern sind. So spricht er $\mathbf{u}$. a. neben der sprachlichen und der logisch-mathematischen Intelligenz von der Bewegungsintelligenz, der musikalischen Intelligenz, der sozialen, praktischen und wissenschaftlichen Intelligenz. Wissenschaftliche Intelligenz wird gefördert, wenn Neugierde geweckt, Experimentieren zugelassen, die Wahrnehmung für Details geschärft wird. Praktische Intelligenz entwickelt sich beim Bauen, Basteln, Konstruieren, beim Werkzeuggebrauch wie Hammer und Säge, beim systematischen Vorgehen zur Problemlösung, beim Verstehen einer Gesamtkonstruktion. In der Zusammenarbeit mit anderen wird die soziale Intelligenz erweitert, eigene Interessen sollen dabei erkannt, Gefühle z. B. bildnerisch ausgedrückt und Humor soll entwickelt werden. Melodien, Farbklänge, Stilrichtungen kennen zu lernen, Bilder anzuschauen, Musik zu hören etc. fördert die bildnerisch-musikalische Intelligenz und das Sprechen über Kunstwerke die sprachliche Intelligenz. Theater zu spielen oder beidhändiges Zeichnen, Rhythmusübungen oder Tanzen entwickelt die Bewegungsintelligenz, das räumliche Gestalten die logisch-mathematische Intelligenz (Gardner 1999).

Kinder kommen heute mit außerordentlich heterogenen körperlichen, sozialen und/oder kognitiven Voraussetzungen in elementare Bildungseinrichtungen. Diese Heterogenität wird nicht nur durch die unterschiedliche kulturelle sowie religiöse Herkunft der Kinder und durch mangelnde Sprachkenntnisse verursacht, sondern auch durch zerrissene Familien, soziale und kulturelle Brüche sowie die Mediatisierung der Kindheit. Hier kann die ästhetische Bildung ausgleichend wirken: Denn z. B. im bildnerischen und musikalischen Tun, in Tanz und Pantomime zeigen sich keine Differenzen in der Sprache, es zeigen sich weder soziale noch religiöse Unterschiede und auch das intellektuelle Vermögen spiegelt sich kaum in den gestalteten Ergebnissen. Die Kinder haben die Möglichkeit, ihre Befindlichkeiten, Träume, Wünsche und Ängste gestalterisch zum Ausdruck zu bringen und, bei Bedarf, darüber zu reden. Die Gestaltgebung im Bild oder in einer Aufführung ist weitgehend unabhängig von Herkunft und familiärer Prägung - und die visuelle Erscheinung des Gestalteten ist ein universell verständliches Kommunikationsmittel, das die Kinder bei aller Heterogenität verbindet.

\section{Alterstypisches bildnerisches Gestalten}

Sukzessiv erobert sich das Kind das Reich der Kritzeleien (Stritzker/Kirchner/Peez 2008). Betrachtet man die oft über Monate hinweg sich wiederholenden Kritzelspuren, lassen sich diverse verschiedene Kritzelzeichen unterscheiden: Es gibt Hiebkritzel, wenn der Stift auf das Papier aufschlägt, und Schwung-, Kreis- und Zickzackkritzel, die der Bewegung folgen, kreuzförmige Ballungen und Verdichtungen, die bereits einen Ausdruckswillen erahnen lassen. Manche Kritzel ergeben sich aus reiner Bewegungslust, Schreibkritzel ahmen das Schreiben der Erwachsenen nach.

Mit zunehmender Übung gelingt es dem Kind die Linien gezielt zu führen: Wenn der erste Kreis entsteht, ist es dem Kind geglückt, den Beginn und das Ende der Linie zusammenzuführen. Mit großem Stolz zeigt das Kind die neue Form, die ein Innen und Außen hat, die gefüllt und weiterentwickelt werden kann. Eine weitere, wie der Kreis mittlerweile aus dem Handgelenk und der Hand gesteuerte Form ist das Kreuz. Die gerichteten Linien weisen einen größtmöglichen Richtungsunterschied auf. Diese Richtungsunterscheidung prägt die Kinderzeichnung noch bis weit ins Grundschulalter hinein. Der Kreis und das im rechten 
Winkel angelegte Kreuz sind die Grundformen frühkindlichen Ausdrucks, die nun in vielerlei Varianten kombiniert werden können. Die meisten Kinder beginnen Ende des ersten Lebensjahres zu schmieren und im zweiten Lebensjahr zu kritzeln, und auch Dreijährige kritzeln noch gerne, zum Teil mit großer Ausdauer - getragen von Freude an der Bewegung, dem Rhythmus, der Farbigkeit (vgl. Kirchner 2008).

Im Alter zwischen drei und sechs Jahren entwickelt das Kind allmählich Zeichen und Formen für die Menschdarstellung. Zunächst entstehen die so genannten Kopffüßler, einigermaßen runde Gebilde mit Zeichen im Inneren und nach außen wegstrebenden Linien. Resultierend aus dem eigenen Körpergefühl und dem emotionalen Verhältnis zur äußeren Welt entsteht eine Bildsprache, die verdeutlicht, dass das Kind erste Erkundungsgänge in die Umwelt unternimmt. Der Kopf steht für den gesamten Mensch, der Mensch ist eine Einheit. Aus dieser Einheit heraus greift das Kind nach außen und stellt sich auf die Beine. Punkte oder Kreise stehen für die Augen. Der Mensch wird allmählich ausdifferenziert mit Haaren, Ohren, Nase, Mund und Füßen. Dabei ist es oftmals unerheblich, von welcher Seite aus eine Figur gezeichnet wird, denn die Kinder zeichnen von allen Seiten, sie drehen das Blatt, es gibt noch kein Oben und Unten.

In der Regel zeigt sich der differenzierter wiedergegebene Mensch in seiner prägnantesten Ansicht: von vorne, mit meist rechtwinklig abstehenden Armen und nach unten gerichteten Beinen. Das Kind fügt sukzessiv die einzelnen Körperteile und Gegenstände zusammen, um die es weiß und die es interessieren. Die einzelnen Bildelemente werden auf einer Standlinie nebeneinander aufgereiht und zunehmend ausgestaltet. Aus einzelnen Strichen werden breite Beine, daran stecken Schuhe, die ebenfalls in ihrer prägnantesten Ansicht, nämlich von der Seite gezeichnet werden. Hände werden detaillierter, es zeigen sich Finger, und die Kinder unterscheiden zwischen Jungen und Mädchen, zwischen Mann und Frau. Entwicklungen wie bei der Menschdarstellung zeigen sich auch bei allen anderen Bildthemen der Kinder: innerhalb der Tierdarstellung, der Pflanzen, der Alltagsgegenstände. Dennoch steht der Mensch zunächst vorrangig im kindlichen Interesse, erst später folgen meist Tiere und Pflanzen. Die Phase bildnerischer Ausdrucksformen im mittleren Kindesalter wird in der Regel als „Schemaphase" gekennzeichnet. Einzelne Bildelemente werden zunehmend an den Flächenkoordinaten orientiert ausgerichtet, und es entwickelt sich eine stabile, gerichtete und relationale Flächenorganisation. So kann sich eine räumliche Anordnung durch das Errichten von vertikalen figurativen Elementen auf einer horizontalen Stand- oder Bodenlinie ergeben. Wertschätzung erfährt das Kind, wenn eine Arbeit präsentiert und besprochen wird. Wenn eine Gestaltungsarbeit nicht geschätzt wird, sind Kinder leicht demotiviert und hören in der Regel auf, von sich aus bildnerisch tätig zu werden.

\section{Ästhetisches Verhalten}

Nicht jedem Kind liegt das Zeichnen, für manches Kind sind die Bewegung und der Tanz ein Weg, z. B. Raumerfahrungen zu klären, andere Kinder benötigen hierfür die Darstellung auf dem Papier. Das Ordnen von Gegenständen zueinander kann ebenso beim Malen wie beim Sammeln stattfinden, und Fantasiegeschichten können sowohl im Baumhaus entwickelt werden wie auf dem Zeichenblock. Kinder bauen sich Räume mit Decken und Tüchern, Kisten und Brettern, sie hämmern, sägen, raspeln, werken und erfinden Spielfiguren, Fahrzeuge, Maschinen und ähnliche Dinge. Bauen ist eine Tätigkeit, die der Aneignung besonders von Raumempfinden und Raumvorstellungen dient, und zwar durch das In-Besitz-Nehmen von 
Raum sowie durch das Gestalten von plastischen Gebilden. Räumlichkeit wird durch die eigene Bautätigkeit erlebbar und verfügbar gemacht. Die Entwicklung der Bautätigkeit von Kindern zeichnet sich durch zunehmend differenzierte Funktionalität und Konstruktionsvielfalt, eine formenreiche Bausprache und durch Raumausdehnung aus. Das Ausprobieren ungewohnter Lösungswege und die Antizipation von Lösungsprinzipien fördern dabei das technische Verständnis. Im Umgang mit Alltagsgegenständen, Abfallprodukten und Naturstoffen steht das entdeckende, fantasiereiche ästhetische Tun im Zentrum des Gestaltens. Kinder möchten ausprobieren, wie Materialien sich verbinden lassen, wie stabil das Holz, die Erde, das Klebeband ist; wie viel Wasser die Farbe verträgt, wann der Bleistift durchbricht oder wie der Computer funktioniert. Sie benötigen diese ästhetischen Erfahrungen, um mit ihrer Umwelt in Kontakt zu treten und diese zu erkunden. Zunehmend mehren sich Untersuchungen, die das kreativitätsfördernde Potenzial einer materialanregenden Umgebung bestätigen (Heyl 2006; Sung-Hiu 2008; Kirchner/Peez 2009).

Das Erkennen von Farben - und damit auch das Benennen - bildet sich erst langsam und mit erheblichen Altersunterschieden bei Kindern heraus. Säuglinge können lediglich zwischen Hell und Dunkel unterscheiden, Farben sehen sie überhaupt nicht. Allmählich wird die Welt farbiger, zunächst werden Rottöne bzw. Rotvarianten wahrgenommen und weitere warme Farben, dann erst die kälteren Töne. Insofern mag es nicht verwundern, dass manche Kinder erst recht spät Farben bewusst wahrnehmen und artikulieren können. Die Farbwahrnehmung lässt sich im Alltag hervorragend üben: Beispielsweise bietet das Beschreiben der Grüntöne in der Natur ein ebenso breites Differenzierungsspektrum wie etwa die Nuancen von Rosarot bis Violett des Abendhimmels. Kinder lieben Farben, sie klecksen und lassen die Farben ineinander laufen, obgleich sie schon lange in der Lage sind, Bildgegenstände darzustellen. Schon mit den Fingern, spätestens wenn sie einen Pinsel halten können, erproben Kinder mit großer Lust den Umgang mit der Farbe, reagieren auf Farbklänge und wachsen langsam in das farbige Gestalten hinein (vgl. Kirchner 2008).

Zeitschriften und Bilderbücher, Kinderspielzeug wie Kunstwerke bieten Anlass, über Bilder zu sprechen. Nicht nur, was zu sehen ist, ist relevant, auch wie es dargestellt wird. Welche Gestaltungsmittel werden eingesetzt, wie ist die Farbwahl, die Bildkomposition, die Malweise? Wird etwas stark übertrieben, karikaturhaft dargestellt, wo liegen die Unterschiede im Ausdruck? Welche Farben werden für abendliche Szenen, welche für den Mittag genutzt? Wir können mit Kindern über Bauwerke, über Mode, Werbung, Zeitungsgrafiken und Vieles mehr sprechen, um sie für die ästhetische Wahrnehmung ihrer Umgebung zu sensibilisieren. Kinder haben eindeutige Präferenzen, wenn es darum geht, Bilderbücher und Illustrationen in Märchen- oder Lesebüchern zu beurteilen. Sie können das Traumhafte oder Plakative, das Kitschige oder Erzählerische in Bezug auf die jeweilige Geschichte unterscheiden und artikulieren.

\section{Aktueller Forschungsstand zur ästhetischen Bildung}

Ästhetische Bildungsprozesse in der frühen Kindheit sind bislang noch wenig erforscht. Dies mag verwundern, denn die Kinderzeichnung wurde in der ersten Hälfte des 20. Jahrhunderts intensiv untersucht; die Ergebnisse mündeten in idealtypische Entwicklungsreihen, die von einer engen Verzahnung kognitiver und zeichnerischer Entwicklung ausgingen. Eine einseitige Kopplung von Kinderzeichnung und Denkentwicklung ist allerdings nicht haltbar: Individuelle Darstellungsformen, Ausdrucksspezifika und Mitteilungsabsichten müssen eben- 
falls berücksichtigt werden. Subjektive Gestaltungstendenzen und persönliche Bedeutungsbezüge prägen die bildnerischen Äußerungen, dies zeigen die wenigen Befunde der zweiten Hälfte des 20. Jahrhunderts. Zunehmend jedoch wächst wieder das Interesse an empirischen Forschungen zur ästhetischen Bildung (vgl. Kirchner/Kirschenmann/ Miller 2010; Kirchner/ Miller 2013). Einige Untersuchungen beziehen sich auf Einzelaspekte wie z. B. das frühe Zeichnen und Schmieren (Stritzker/Peez/Kirchner 2008), andere untersuchen die Bildrezeption (Kirchner 1999; Uhlig 2005), mögliche Bildpräferenzen (EU-Projekt 2010), das Malen (Dietl 2004) oder Experimentieren (Reuter 2007) im Grundschulalter. Zur ästhetischen Erfahrung in der Kindheit legen Mattenklott und Rora (2004) eine Publikation vor, in deren Zentrum steht, was Kinder im Umgang mit Musik, Kunst oder Literatur empfinden und erleben. Neben dem Diskurs über ästhetische Phänomene werden anhand von Kindertexten und Beispielen bildnerischer Tätigkeit Dimensionen ästhetischer Erfahrung aufgezeigt. Die meisten Forschungen beziehen sich auf das ästhetische Verhalten in der mittleren Kindheit. Mit der frühkindlichen ästhetischen Bildung befasst sich die Arbeitsgruppe Grundschule im BDK (Fachverband für Kunstpädagogik e.V. 2009), um die Charakteristika ästhetischer Bildung $\mathrm{zu}$ fassen und daraus Handlungsoptionen für die Pädagoginnen und Pädagogen zu entwickeln. Entstanden ist eine anschauliche Schilderung dessen, was ästhetische Prozesse im Kindesalter ausmacht, wie diese verlaufen könnten und wann es sinnvoll ist, einzugreifen, zu fördern, Materialien anzubieten, Gestaltungsfreiraum zu lassen, Gestaltungsprozesse zu intensivieren. Die Prozesse frühkindlichen ästhetischen Lernens werden in ihren unterschiedlichen Dimensionen sehr ausführlich mit Bezug auf verschiedene ästhetische Praxisfelder in einem Handbuch für elementare Bildung beschrieben (Duncker et al. 2010). Rittelmeyer (2007) versammelt Argumente für eine humanistisch-ästhetische Bildung, die er mit empirischen Befunden und Erkenntnissen aus der Hirnforschung belegt, und gibt mit seinem Band „Warum und wozu ästhetische Bildung" (2010) einen Forschungsüberblick über die Transferwirkungen künstlerischer Tätigkeiten.

Entscheidende Impulse für die empirische Bildungsforschung in der Kunstpädagogik sind den Publikationen von Georg Peez $(2000 ; 2007)$ zu verdanken. Zu erwähnen ist auch die Studie von Monika Miller (2013), die Indikatoren bildnerischer Fähigkeiten bei Kindern filtert. Die Befunde zeigen, dass verschiedene, zum Teil sehr unterschiedliche Kompetenzen das bildnerische Vermögen von Kindern bestimmen: exakte Beobachtung, präzise Wahrnehmung, vielfaches Abzeichnen, fantasievolles Ideen entwickeln, das Interesse an ornamentalen Darstellungsformen, aber auch Neugierde, motorische Geschicklichkeit, Leidenschaft und Ausdauer, hinzu kommen die Vorstellungsfähigkeit, wie etwa Erinnerungsbilder zu imaginieren, und kombinierbare Vorstellungen zu entwickeln, das Formgedächtnis oder das Wissen um die abzuzeichnenden Gegenstände.

Aus bildungstheoretischer Perspektive beleuchtet die von Gert E. Schäfer und Roswitha Staege (2010) herausgegebene Publikation Bildungsprozesse von Kindern als Akteuren in einer gesellschaftlich determinierten Welt. Hierbei wird insbesondere lokalen kulturellen Rahmenbedingungen Aufmerksamkeit geschenkt. Die ethnographischen Studien zielen auf pädagogische Felder, in denen sich das Lernen entwickelt. In Bezug auf die ästhetischen Bildungsprozesse ist die empirische Studie von Roswitha Staege (2010) interessant, die versucht, ästhetische Erfahrung anhand videographisch erfasster Daten interpretativ zu rekonstruieren. Dabei stehen musikalische Tätigkeiten von Kindergartenkindern im Vordergrund, die anhand von Fallbeispielen exemplarisch interpretiert werden.

Dass die ästhetische Bildung über Materialerfahrung, Körpererfahrung und den gestalterischen Ausdruck die Ich-Identität stärkt und dem Selbstverlust in virtuellen Welten mit sinnlich gegründeter Bodenhaftung entgegenwirkt, daran besteht kein Zweifel. Anschaulich belegen einige Studien aus kunsttherapeutischem Terrain die positive Wirkung gestalterischen 
Tuns. Dahinter steht die Annahme, dass Kinder und Jugendliche sich mit bildnerischen Verfahren unmittelbarer und direkter ausdrücken können als mit Worten. Unaussprechliches, aber auch Wünsche, Träume und Hoffnungen können nonverbal zum Vorschein kommen und damit kommunizierbar werden. Eine meist negative Erfahrung verliert ihre Singularität, sie kann geteilt und miteinander besprochen werden. Zugleich trägt der Prozess bildnerischen Gestaltens dazu bei, spielerisch und unkompliziert vorhandene Ressourcen zu aktivieren und Selbstheilungskräfte zu fördern. Die von Harald Gruber und Barbara Wichelhaus (2011) herausgegebene Publikation zur Kunsttherapie mit Kindern zeigt, inwiefern Störungen diagnostiziert und behandelt werden können, dass die Kinderzeichnung ein wesentlicher Indikator in kunsttherapeutischen Prozessen sein kann oder wie Ich-Stärke auf der Basis komplexer psycho-physischer Prozesse gefördert werden kann. Eine empirische Studie zu den Wirkfaktoren künstlerischer Therapien belegt, dass insbesondere die Anregung schöpferischer Prozesse, die Stimulation aktiver Gestaltungsfähigkeit und die Unterstützung des nonverbalen Ausdrucks als entscheidende Kriterien für die Wirksamkeit ästhetischer Bildungsprozesse bestätigt werden können (ebd. 2011).

\section{Literatur}

Arbeitsgruppe Grundschule im BDK - Fachverband für Kunstpädagogik e.V. (2009): Frühkindliche ästhetische Bildung - ein Diskussionsbeitrag, Hannover

Dietl, M.-L. (2004): Kindermalerei. Zum Gebrauch der Farbe am Ende der Grundschulzeit, Münster

Duncker, L./Lieber, G./Neuss, N./Uhlig, B. (Hrsg.) (2010): Bildung in der Kindheit. Das Handbuch zum Lernen in Kindergarten und Grundschule, Seelze

EU-Projekt imago (2010): Lernen mit Bildern. Anregungen zur Arbeit im Vor- und Grundschulalter, o.O.

Gardner, H. (1999): Kreative Intelligenz. Was wir mit Mozart, Freud, Woolf und Gandhi gemeinsam haben, Frankfurt/M.

Gruber, H./Wichelhaus, B. (Hrsg.) (2011): Kunsttherapie mit Kindern und Jugendlichen. Aktuelle Bezüge aus klinischen und sozialen Anwendungsfeldern, Berlin

Heyl, T. (2006): Schule der Phantasie. Dissertation. Universität Koblenz-Landau, Campus Landau

Kirchner, C. (1999): Kinder und Kunst der Gegenwart: Zur Erfahrung mit zeitgenössischer Kunst in der Grundschule, (1. Aufl.), Seelze

Kirchner, C./Dietl, M.-L. Dietl (Hrsg.) (2002): Bildnerisch gestalten in der Grundschule. Kunst+Unterricht Sammelband, Seelze-Velber

Kirchner, C. (2008): Kinder \& Kunst. Was Erwachsene wissen sollten, Seelze-Velber

Kirchner, C./Kirschenmann, J./Miller, M. (Hrsg.) (2010): Kinderzeichnung und jugendkultureller Ausdruck. Forschungsstand - Forschungsperspektiven, München

Kirchner, C./Miller, M. (2013): Neue Forschungsperspektiven auf die Entwicklung und Förderung der Bildsprache. In: Schulz, Frank/ Seumel, Ines (Hg.): U20 Kindheit Jugend Bildsprache. München 2013, S. 332-344

Kirchner, C./Peez, G. (2009): Praxis Pädagogik: Kreativität in der Grundschule erfolgreich fördern, Braunschweig

Mattenklott, G./Rora, C. (Hrsg.) (2004): Ästhetische Erfahrung in der Kindheit. Theoretische Grundlagen und empirische Forschung, Weinheim/München

Miller, M. (2013): Zeichnerische Begabung. Indikatoren im Kindes- und Jugendalter, München

Neuenschwander, M. P. (1996): Entwicklung und Identität im Jugendalter, Bern/Stuttgart/Wien

Peez, G. (2000): Qualitative empirische Forschung in der Kunstpädagogik. Methodologische Analysen und praxisbezogene Konzepte zu Fallstudien über ästhetische Prozesse, biografische Aspekte und soziale Interaktion in unterschiedlichen Bereichen der Kunstpädagogik, Hannover 
Peez, G. (Hrsg.) (2007): Handbuch Fallforschung in der Ästhetischen Bildung/Kunstpädagogik. Qualitative Empirie für Studium, Praktikum, Referendariat und Unterricht, Baltmannsweiler

Reuter, O. M. (2007): Experimentieren. Ästhetisches Verhalten von Grundschulkindern. München

Rittelmeyer, Ch. (2007): Kindheit in Bedrängnis. Zwischen Kulturindustrie und technokratischer Bildungsreform, Stuttgart

Rittelmeyer, Ch. (2010): Warum und wozu ästhetische Bildung? Über Transferwirkungen künstlerischer Tätigkeiten. Ein Forschungsüberblick, Oberhausen

Schäfer, G. E. (Hrsg.) (2004): Bildung beginnt mit der Geburt, Weinheim/Basel

Schäfer, G. E./Staege, R. (Hrsg.) (2010): Frühkindliche Lernprozesse verstehen. Ethnographische und phänomenologische Beiträge zur Bildungsforschung, Weinheim/München

Staege, R. (2011): How to do things with music. Dokumentarische Videointerpretation als Zugang zur ästhetischen Erfahrung von Kindern. In: Gruber, H./Wichelhaus, B. (Hrsg.): Kunsttherapie mit Kindern und Jugendlichen. Aktuelle Bezüge aus klinischen und sozialen Anwendungsfeldern, Berlin

Stritzker, U./Peez, G./Kirchner, C. (2008): Frühes Schmieren und erste Kritzel - Anfänge der Kinderzeichnung, Norderstedt

Sung-Hui, K. (2008): Kreativitätsförderung und Montessori-Pädagogik. Untersuchungen bei Kindern im Vorschulalter, Berlin

Uhlig, B. (2005): Kunstrezeption in der Grundschule. Zu einer grundschulspezifischen Rezeptionsmethodik, München

Wichelhaus, B. (1995): Kompensatorischer Kunstunterricht. In: Kunst + Unterricht 191/1995, S. 35-39 